\title{
Frequency-Dependent Changes of Spontaneous Low-Frequency Fluctuations in Patients with Age- Related Macular Degeneration
}

Feijia Xu ( $\sim$ salemkevin@hotmail.com )

Shanghai Tenth People's Hospital https://orcid.org/0000-0001-9450-0513

Ping Lin

Shanghai Tenth People's Hospital

Xiaolian Su

Shanghai Tenth People's Hospital

Qing Peng

Shanghai Tenth People's Hospital

Guangyu Tang

Shanghai Tenth People's Hospital https://orcid.org/0000-0003-4181-3318

\section{Research Article}

Keywords: Age-related macular degeneration, low-frequency fluctuations, ALFF, fALFF, PerAF

Posted Date: February 28th, 2022

DOI: https://doi.org/10.21203/rs.3.rs-1376251/v1

License: (9) This work is licensed under a Creative Commons Attribution 4.0 International License. Read Full License 


\section{Abstract}

Age-related macular degeneration (AMD) is the leading cause of irreversible blindness among older adults. AMD affects the patient's ability to read, write and recognize faces. Prior neuroimaging studies revealed abnormal functional connectivity within visual network and default mode network (DMN). 25 diagnosed AMD patients and 25 age, sex matched healthy controls were recruited into our study for resting-state functional magnetic resonance imaging (rs-fMRI) scans. Three different low frequency fluctuations metrics including amplitude of low-frequency fluctuation (ALFF), fractional ALFF (fALFF), and percent amplitude of fluctuation (PerAF) were introduced to detect and analyze spontaneous brain activity between $\mathrm{AMD}$ and $\mathrm{HCs}$. For each metrics, traditional frequency band $(0.01-0.08 \mathrm{~Hz})$, slow-4 frequency band $(0.027-0.073 \mathrm{~Hz})$ and slow- 5 frequency band $(0.01-0.027 \mathrm{~Hz})$ were examined respectively. Compared to HCs group, our result revealed that significant difference in ALFF, fALFF and PerAF showed in several brain regions including orbital frontal gyrus, fusiform gyrus, amygdala, precuneus, angular gyrus, median cingulate gyrus and rectus gyrus in AMD group at traditional frequency band. Moreover, findings of the present study demonstrated that abnormal spontaneous brain activity of fALFF in AMD is frequency-dependent, and may provide a new perspective to explain the pathological mechanism of AMD.

\section{Introduction}

Age-related macular degeneration (AMD) is a complex eye disease, characterized by progressive central vision impairment due to degenerative or neovascular changes in the macular region of the retina (Blasiak, 2020; Gehrs, Anderson, Johnson, \& Hageman, 2006). Due to the lack of effective treatment for the majority of AMD patients, AMD is the leading cause of irreversible blindness among older adults, particularly aged 60 to 85 years, further results in patients' inability to read, write, recognize faces, and perform physical activities that requiring normal vision (van Leeuwen, Klaver, Vingerling, Hofman, \& de Jong, 2003). In addition to being cause of visual impairment, AMD is often associated with Alzheimer's disease (AD) and depression based on previous studies (R. J. Casten, Rovner, \& Tasman, 2004; Kaarniranta, Salminen, Haapasalo, Soininen, \& Hiltunen, 2011; Lee et al., 2019). Therefore, exploring the underlying pathological mechanisms and finding reliable neuroimaging markers is crucial for the treatment of AMD.

Resting-State Functional Magnetic Resonance Imaging (rs-fMRI) of blood oxygenation level dependent (BOLD), as a non-invasive neuroimaging technique, has been applied for clinical research to study abnormal brain function, and has provided novel insights into the pathological mechanism of various diseases (Puig et al., 2018; Raichle \& Gusnard, 2005; Sheline \& Raichle, 2013; Workman et al., 2017). Previous rs-fMRI study using independent component analysis (ICA) approach showed that AMD patients exhibited reduced functional connectivity (FC) within visual networks (Zhuang et al., 2018). Another study based on regions of interest (ROIs) approach, exhibited stronger FC in phonemic fluency-related brain regions and default mode network (DMN) in AMD patients (Whitson et al., 2015). Evidences from brain network in response to decreased visual input have revealed abnormalities of FC in patients suffering 
from AMD. However, they unable to precisely provide the brain regions that produce abnormal spontaneous brain activity. Investigation of specific abnormal brain regions in patients with AMD is important for the clinical researches and applications. It has been reported that spontaneous lowfrequency $(0.01-0.08 \mathrm{HZ}$ ) fluctuations (LFFs) of BOLD signal might contain physiological information (Biswal, Yetkin, Haughton, \& Hyde, 1995), and are closely linked to spontaneous neuronal activity (Logothetis, Pauls, Augath, Trinath, \& Oeltermann, 2001; Raichle \& Gusnard, 2005).

Several rs-fMRI metrics are available for measuring the regional intensity of spontaneous LFFs. Amplitude of low-frequency fluctuation (ALFF) (Zang et al., 2007), can directly reflect the intensity of spontaneous neural activity and has been applied widely in eye diseases including glaucoma (Peng et al., 2021), strabismus (Min et al., 2018), retinal vein occlusion (Wu et al., 2019), and hypertensive retinopathy (Hu et al., 2021). ALFF has also been proven to have moderate to high test-retest reliability primarily in gray matter (Zou et al., 2008). Based on ALFF, Zuo et al. proposed fractional ALFF (fALFF) approach, which is defined as the total power within the low-frequency range $(0.01-0.08 \mathrm{~Hz})$ divided by the total power in the entire detectable frequency range (Zou et al., 2008). These two metrics characterize the LFFs from different aspects: ALFF reflects the intensity or strength of LFFs, while fALFF represents the relative contribution of specific LFFs to the whole detectable frequency range (Zuo et al., 2010). Another widely applied method measuring the characteristics of spontaneous brain activity is percent amplitude of fluctuation (PerAF). Unlike ALFF and fALFF, PerAF calculates the percent BOLD fluctuations relative to the average BOLD signal strength at each time point and averaging the whole time series that directly reflects the BOLD signal fluctuation in the resting state (Jia et al., 2020), and it represents the percentage of signal alteration. Previous studies have revealed that PerAF might have higher test-retest reliability than both ALFF and fALFF (Jia et al., 2020; Zhao et al., 2018). Therefore, PerAF is also a promising method for rs-fMRI signals detection. The combination of the three metrics could characteristic the brain spontaneous activities of AMD patients in an all-round way.

However, most rs-fMRI studies adopting the methods above investigated LFFs amplitude at traditional frequency band $(0.01-0.08 \mathrm{~Hz}$ ) (Hu et al., 2021; Min et al., 2018; Peng et al., 2021), because this frequency band was thought to be related to neuronal fluctuations (Biswal et al., 1995; D. Zhang \& Raichle, 2010). However, some studies have observed linear distribution of neuronal oscillations on the scale of natural logarithmic and the signal within independent frequency bands are produced by distinct oscillators with specific properties and physiological functions (Buzsáki \& Draguhn, 2004; Penttonen \& Buzsáki, 2003). According to Buzsaki and Draguhn, LFFs frequency ranges could be typically subdivided into 4 frequency bands: slow- 5 frequency band $(0.01-0.027 \mathrm{~Hz})$, slow- 4 frequency band $(0.027-0.073 \mathrm{~Hz})$, slow-3 frequency band $(0.073-0.198 \mathrm{~Hz})$ and slow-2 frequency band $(0.198-0.25 \mathrm{~Hz})$ (Buzsáki \& Draguhn, 2004; Zuo et al., 2010). Slow-4 and slow-5 frequency bands are most closely related to gray matter signal and are useful in identifying brain functional disorders (Zuo et al., 2010). Previous Study found that ALFF in schizophrenia patients for slow-4 frequency band were higher in cingulate cortex, fusiform gyrus, basal ganglia, and midbrain, compared to ALFF in slow-5 (R. Yu et al., 2014). Another study showed that aMCI patients had greatly lower ALFF and fALFF values in the posterior cingulate cortex (PCC) and precuneus (PCu) at slow-5 frequency band than at slow-4 frequency band (Han et al., 
2011). These studies indicated frequency-dependent changes were found in specific brain regions, and thus may contribute to further exploration of the mechanism of brain function.

In our study, we compared the changes of LFFs amplitudes between AMD patients and healthy controls (HCs) using three metrics: ALFF, fALFF and PerAF. For each amplitude metrics, traditional frequency band, slow-4 frequency band and slow-5 frequency band were applied separately. Our purpose was to determine 1) whether AMD patients showed abnormalities of LFFs amplitudes in specific brain regions. 2) whether the abnormal spontaneous intrinsic neural activity was associated with specific frequency band.

\section{Methods}

\section{Participants}

Fifty participants were consecutively recruited into the study, of whom 25 diagnosed AMD patients from the Ophthalmology Department of Shanghai Tenth People's Hospital affiliated to Tongji University, and 25 HCs with matched age, gender, and education status comparing to the AMD group. Exclusion criteria: (1) patients with other eye diseases (cataract, glaucoma, optic neuritis, etc.). (2) any evidence of psychiatric disease or neurological diseases. All the HCs in this study met the subsequent criteria: (1) no ocular disease history; (2) no psychiatric disease or neurological diseases; (3) being capable of undergoing MRI examination (no cardiac pacemaker or implanted metal devices, etc.).

Best-corrected visual acuity (BCVA) in Logarithm of the Minimum Angle of Resolution (LogMAR) table, as the measurement of the best vision correction that can be achieved by glasses or contact lenses, was applied to measure visual acuity of both eyes for each participant. To evaluate cognitive ability of each participant, two routine cognitive screening scales including Mini-Mental State Examination (MMSE) and Montreal Cognitive Assessment (MoCA) were utilized, in which MoCA is more sensitive for mild cognitive impairment than MMSE (S. Zhang et al., 2021).

\section{Ethical approval}

was given by hospital's medical ethics committee in line with the Declaration of Helsinki. All participants signed informed consents after informing of the purposes, contents, and potential risks.

\section{MRI Acquisition}

MRI data were acquired on a 3-Tesla MRI scanner (Philips Medical Systems, Best, The Netherlands) at Shanghai Tenth People's Hospital. All participants were asked to keep their eyes opened and stay awake. Brain structural MRI data was obtained using a T1-weighted 3D turbo field echo sequence for the whole brain with repetition time $=6.0 \mathrm{~ms}$, echo time $=2.7 \mathrm{~ms}$, field of view $=230 \mathrm{~mm} \times 230 \mathrm{~mm}$, voxel size $=0.9$ $\times 0.9 \times 0.9 \mathrm{~mm}$, flip angle $=8^{\circ}$, slices $=200$, slice orientation $=$ sagittal, scan duration time $=4$ min and 42 seconds. The rs-fMRI data were 240 volumes (scanning time $=8 \mathrm{~min}$ ) of echo planar imaging (EPI) 
sequence with the following parameters: repetition time $=2,000 \mathrm{~ms}$, echo time $=35 \mathrm{~ms}$, voxel size $=3.6 \times$ $3.6 \times 3.6 \mathrm{~mm}$, field of view $=250 \mathrm{~mm} \times 250 \mathrm{~mm}$, slices $=35$, thickness $/ \mathrm{gap}=3.6 / 0 \mathrm{~mm}$.

\section{MRI data preprocessing}

All functional imaging data processing were conducted by Statistical Parametric Mapping 12 (SPM12, http://www.fil.ion.ucl.ac.uk/spm/software/spm12) and RESTplus (restplus_v1.25, http://restfmri.net/forum/restplus) toolkits implemented in MATLAB 2017b (MathWorks, Natick, MA, USA) (Jia et al., 2020). Briefly, it included following steps. The first 10 time points were removed for the reduction of the non-equilibrium effects of magnetization. The remaining 230 scans of each participant underwent slice timing correction, realignment, co-registration of the high-resolution structural images to the mean functional image. Then, the images were normalized spatially to the Montreal Neurological Institute (MNI) template in new segment and resampled to $3 \times 3 \times 3 \mathrm{~mm}^{3}$. Any participant with head motion of $>3 \mathrm{~mm}$ or translation rotation $>3^{\circ}$ in any direction was removed. Then, we used a Gaussian kernel of $6 \mathrm{~mm}$ full-width at half-maximum to smoothen the images. Finally, detrending was performed for the elimination of linear drift. For reduction of physiological noise, the signals of white matter (WM), cerebrospinal fluid (CSF), Friston-24 parameters model of head motion were regressed as covariates (Friston, Williams, Howard, Frackowiak, \& Turner, 1996).

\section{Calculation of rs-fMRI Metrics}

In our study, ALFF, fALFF and PerAF calculation were performed using RESTplus software at three different frequency bands: traditional frequency band $(0.01-0.08 \mathrm{~Hz})$, slow- 5 frequency band $(0.01-$ $0.027 \mathrm{~Hz}$ ), and slow-4 frequency band $(0.027-0.073 \mathrm{~Hz})$, based on previous studies (Buzsáki \& Draguhn, 2004).

After preprocessing, Fast Fourier Transformation (FFT) was used to convert the time series for each voxel to the frequency domain, then we obtained the power spectrum. Second, we calculated the square root of the power spectrum at each frequency. Third, the square root was averaged across a predefined frequency range at each voxel, and ALFF was obtained. The fALFF is calculated as a ratio of the ALFF within a predefined frequency range to the entire frequency band. Each voxel's ALFF/fALFF was divided by the global mean ALFF/fALFF value for standardization.

PerAF was obtained by two steps. Firstly, the percentage of BOLD signal intensity relative to the mean BOLD signal intensity for each time point was measured. Then, we calculated the average across the whole time series. The PerAF of each voxel was also divided by the global mean PerAF value to reduce the global effects of variability.

\section{Statistical analysis}

First, demographic and the clinical characteristics including age, sex, BCVA of both eyes, MoCA scores, MMSE scores, duration time and education status of AMD were imported into the SPSS 20.0 software (IBM, Armonk, NY) for comparison between AMD group and HCs. 
Then, two-sample $t$-tests was performed to compare the ALFF, fALFF and PerAF maps between AMD group and $\mathrm{HCs}$ at three different frequency bands respectively. The multiple comparison correction was based on the Gaussian Random Field (GRF) theory with a voxel-level $P<0.01$ and cluster-level $P<0.05$.

\section{Results}

\section{Demographic and Clinical Characteristics}

The clinical characteristics of the AMD and HCs group including BCVA, MMSE scores, MoCA scores, education status and duration time of AMD were showed in Table 1. No significant differences in age, gender, education status and MMSE scores were observed between the AMD and HCs group. However, the AMD group exhibited relatively lower MoCA scores than HCs group $(P<0.001)$. The significant differences were obtained in BCVA-right $(P<0.001)$ and BCVA-left $(P<0.001)$ between the two groups.

Table 1

Demographic and Clinical Characteristics

\begin{tabular}{|c|c|c|c|c|}
\hline Conditions & AMD & HCs & $T$-value $/ \chi^{2}$ & $P$-value \\
\hline Age (years) & $71.6 \pm 1.776$ & $68.64 \pm 1.362$ & 1.322 & $0.192^{\mathrm{a}}$ \\
\hline Sex (Male/Female ) & $14 / 11$ & $11 / 14$ & 0.837 & $0.406^{\mathrm{b}}$ \\
\hline MoCA & $24.12 \pm 0.570$ & $27.16 \pm 0.275$ & -4.807 & $\varangle 0.001^{\mathrm{a}}$ \\
\hline MMSE & $26.48 \pm 0.425$ & $27.28 \pm 0.319$ & -1.506 & $0.139^{a}$ \\
\hline BCVA_R & $0.488 \pm 0.0477$ & $0.976 \pm 0.0328$ & -8.427 & $\nabla 0.001^{\mathrm{a}}$ \\
\hline BCVA_L & $0.536 \pm 0.0472$ & $0.952 \pm 0.0366$ & -6.966 & $\otimes 0.001^{\mathrm{a}}$ \\
\hline Duration ( years ) & $7.2 \pm 1.133$ & $\mathrm{~N} / \mathrm{A}$ & 6.356 & $\nabla 0.001^{\mathrm{a}}$ \\
\hline Education (years ) & $9.92 \pm 0.562$ & $10.32 \pm 0.613$ & -0.481 & $0.633^{a}$ \\
\hline
\end{tabular}

\section{ALFF Analysis}

As shown in Fig. $1 \mathrm{~A}$ and Table 2, at traditional frequency band, the AMD group showed increased ALFF in right fusiform gyrus and decreased ALFF in left orbital part of inferior frontal gyrus, compared to HCs (voxel $P<0.01$, cluster $P<0.05$, GRF corrected). 
Table 2

Brain regions with significant difference in ALFF at each frequency band between AMD group and HCs

\begin{tabular}{|c|c|c|c|c|c|c|c|}
\hline \multirow[t]{2}{*}{ Brain regions (AAL) } & \multirow[t]{2}{*}{$R / L$} & \multirow[t]{2}{*}{ Voxels } & \multicolumn{3}{|c|}{ MNI coordinates } & \multirow[t]{2}{*}{ BA } & \multirow[t]{2}{*}{$T$-value } \\
\hline & & & $x$ & $y$ & z & & \\
\hline \multicolumn{8}{|c|}{ Traditional frequency band } \\
\hline Fusiform & $\mathrm{R}$ & 178 & 33 & -72 & -12 & 19 & 4.0353 \\
\hline Frontal_Inf_Orb & L & 178 & -24 & 30 & -9 & 11 & -4.025 \\
\hline \multicolumn{8}{|c|}{ Slow-4 frequency band } \\
\hline Temporal_Inf & $\mathrm{R}$ & 161 & 54 & -57 & -12 & 37 & 4.2498 \\
\hline \multicolumn{8}{|c|}{ Slow-5 frequency band } \\
\hline Rectus & L & 209 & -18 & 15 & -12 & 11 & -4.1759 \\
\hline
\end{tabular}

In addition, as shown in Fig. 1B, 1C and Table 2, the AMD group showed increased ALFF in right inferior temporal gyrus at slow-4 frequency band, whereas these patients showed decreased ALFF in left rectus gyrus at slow- 5 frequency band, compared to HCs (voxel $P<0.01$, cluster $P<0.05$, GRF corrected).

\section{fALFF Analysis}

As shown in Fig. 2A and Table 3, at traditional frequency band, the AMD group showed deceased fALFF in right amygdala and increased fALFF in left PCu, left angular gyrus (AG) and left median cingulate gyrus, compared to HCs (voxel $P<0.01$, cluster $P<0.05$, GRF corrected). 
Table 3

Brain regions with significant difference in fALFF at each frequency band between AMD group and HCs.

\begin{tabular}{|c|c|c|c|c|c|c|c|}
\hline \multirow[t]{2}{*}{ Brain regions (AAL) } & \multirow[t]{2}{*}{$R / L$} & \multirow[t]{2}{*}{ Voxels } & \multicolumn{3}{|c|}{ MNI coordinates } & \multirow[t]{2}{*}{ BA } & \multirow[t]{2}{*}{$T$-value } \\
\hline & & & $\mathbf{x}$ & y & $\mathbf{z}$ & & \\
\hline \multicolumn{8}{|c|}{ Traditional frequency band } \\
\hline Amygdala & $\mathrm{R}$ & 55 & 30 & 0 & -18 & 6 & -4.2754 \\
\hline Precuneus & $\mathrm{L}$ & 109 & -3 & -66 & 33 & $\mathrm{~N} / \mathrm{A}$ & 5.356 \\
\hline Angular & $\mathrm{L}$ & 227 & -42 & -66 & 36 & 39 & 4.8804 \\
\hline Cingulum_Mid & $\mathrm{L}$ & 62 & -3 & -36 & 45 & $\mathrm{~N} / \mathrm{A}$ & 5.0054 \\
\hline \multicolumn{8}{|c|}{ Slow-4 frequency band } \\
\hline Fusiform & $\mathrm{R}$ & 73 & 21 & -45 & -12 & 30 & -3.7486 \\
\hline Supra_Marginal & $\mathrm{L}$ & 246 & -57 & -45 & 36 & 40 & 5.0928 \\
\hline Cingulum_Mid & $\mathrm{L}$ & 62 & -3 & -36 & 45 & $\mathrm{~N} / \mathrm{A}$ & 4.7206 \\
\hline \multicolumn{8}{|c|}{ Slow-5 frequency band } \\
\hline Precuneus & $\mathrm{L}$ & 209 & -9 & -57 & 27 & 23 & 5.0873 \\
\hline
\end{tabular}

Additionally, as shown in Fig. 2B, 2C and Table 3, at slow-4 frequency band, the AMD group exhibited decreased fALFF in right fusiform gyrus and increased fALFF in left supramarginal gyrus and left median cingulate gyrus, whereas at slow- 5 frequency band, the AMD group exhibited decreased fALFF in left PCu, compared to HCs (voxel $P<0.01$, cluster $P<0.05$, GRF corrected).

\section{PerAF Analysis}

As shown in Fig. 3A and Table 4, at traditional frequency band, the AMD group showed increased PerAF in right fusiform gyrus and decreased PerAF in left orbital part of middle frontal gyrus and left rectus gyrus, compared to HCs (voxel $P<0.01$, cluster $P<0.05$, GRF corrected). 
Table 4

Brain regions with significant difference in PerAF at each frequency band between AMD patients and HCs.

\begin{tabular}{|c|c|c|c|c|c|c|c|}
\hline \multirow[t]{2}{*}{ Brain regions (AAL) } & \multirow[t]{2}{*}{$R / L$} & \multirow[t]{2}{*}{ Voxels } & \multicolumn{3}{|c|}{ MNI coordinates } & \multirow[t]{2}{*}{ BA } & \multirow[t]{2}{*}{$T$-value } \\
\hline & & & $\mathbf{x}$ & $y$ & $z$ & & \\
\hline \multicolumn{8}{|c|}{ Traditional frequency band } \\
\hline Rectus & $\mathrm{L}$ & 350 & -3 & 60 & -21 & 11 & -5.0733 \\
\hline Fusiform & $\mathrm{R}$ & 290 & 36 & -69 & -15 & 19 & 4.0441 \\
\hline Frontal_Med_Orb & $\mathrm{L}$ & 165 & 0 & 45 & -12 & 11 & -3.5634 \\
\hline \multicolumn{8}{|c|}{ Slow-4 frequency band } \\
\hline Rectus & $\mathrm{L}$ & 322 & -6 & 60 & -21 & 11 & -4.8818 \\
\hline Temporal_Inf & $\mathrm{R}$ & 251 & 54 & -57 & -15 & 37 & 4.085 \\
\hline \multicolumn{8}{|c|}{ Slow- 5 frequency band } \\
\hline Olfactory & L & 122 & -6 & 9 & -18 & 25 & -4.3401 \\
\hline Frontal Sup Orb & L & 179 & -21 & 60 & -15 & 11 & -4.1692 \\
\hline Fusiform & $\mathrm{R}$ & 270 & 36 & -69 & -15 & 19 & 4.5273 \\
\hline
\end{tabular}

Additionally, as shown in Fig. 3B, 3C and Table 4, the AMD group showed increased PerAF in right inferior temporal gyrus and deceased PerAF in left rectus gyrus at slow-4 frequency band, whereas these patients showed decreased PerAF in left olfactory cortex and left orbital part of superior frontal gyrus, and increased PerAF in right fusiform gyrus at slow- 5 frequency band, compared to HCs (voxel $P<0.01$, cluster $P<0.05$, GRF corrected).

\section{Discussion}

In our study, three different rs-fMRI metrics were applied to detect and analyze amplitudes of LFFs in patients with AMD. Compared to HCs group, our result showed significant difference in ALFF, fALFF and PerAF respectively at traditional frequency band in AMD group. Overall, the patients with AMD showed abnormal spontaneous intrinsic brain activity in several brain regions including orbital frontal gyrus, fusiform gyrus, amygdala, $\mathrm{PCu}, \mathrm{AG}$, median cingulate gyrus and rectus gyrus. Moreover, slow- 4 frequency band in fALFF exhibited a wider range of brain regions with abnormal neuronal activity than slow- 5 frequency band did. Therefore, our result indicated that abnormal spontaneous brain activity of fALFF in AMD is frequency-dependent .

\section{ALFF, ALFF and PerAF at traditional frequency band}


Decreased ALFF were observed in left orbital inferior frontal gyrus in AMD patients. In addition, decreased PerAF were showed in left orbital part middle frontal gyrus and left rectus gyrus, compared to HCs. Our results are similar to a study regarding late-on depression patients which also reported deceased ALFF in left middle orbitofrontal gyrus (Yue, Jia, Hou, Zang, \& Yuan, 2015). Besides, previous studies showed that rectus gyrus and orbital frontal gyrus demonstrated deceased gray matter volume in elderly depression and indicated that these region may involve in the neuropathological mechanism of depression (Ballmaier et al., 2004; S. Yu et al., 2018). Growing evidences indicated that prevalence of depression among patients with AMD was relatively higher and total depression scores were strongly associated with visual acuity severity level (Augustin et al., 2007; R. Casten \& Rovner, 2008; Dawson, Mallen, Gouldstone, Yarham, \& Mansell, 2014). Therefore, we speculated that the decreased brain activity in rectus gyrus and orbital frontal gyrus may represent a biomarker for depression. The most likely explanation is that deficits in physical functioning associated with impaired vision due to AMD may attribute to depression (R. J. Casten et al., 2004). In addition, it is necessary for ophthalmologists to implement the depression screening procedures for AMD patients, and refer diagnosed patients to psychiatrists for proper assessment and treatment.

Increased fALFF in left PCu was demonstrated in AMD group, compared to HCs. PCu located on the posteromedial portion of the parietal lobe has involved visuo-spatial imagery (Cavanna \& Trimble, 2006). Besides, PCu also played an important role in episodic memory encoding and retrieval to spatial memory (Frings et al., 2006), and furthermore, it's an important component of DMN (Mak et al., 2017). All above studies indicated PCu play a specific role in higher-order cognitive functions. In contrast to our results, studies of cognitive impairment diseases including $A D$ and $\mathrm{MCl}$ exhibited reduced regional activity of $\mathrm{PCu}$ in ALFF and fALFF (Han et al., 2011; Liu et al., 2014). Further, AMD patients exhibited stronger FC within the DMN, compared with HCs in a rs-fMRI study (Whitson et al., 2015). Therefore, increased regional activity in PCu possibly reflect an analogous potential compensatory changes to support cognitive performance.

Similar to previous rs-fMRI studies on patients with visual disorders including optic neuritis and corneal ulcer, our result also showed increased ALFF and PerAF in right fusiform gyrus (Huang et al., 2015; Shi et al., 2019). It has been revealed that fusiform gyrus is an important brain region involved in the visual cognitive function of facial recognition (Caspers et al., 2015). Therefore, our findings are consistent with the hypothesis that impaired vision caused by AMD affect patients' facial recognition ability. In addition to this, previous study suggested that long-term visual impairment might cause weakening intrinsic connectivity strength of visual cortex (Zhuang et al., 2018). Thus, we speculated that increased neural activity of right fusiform gyrus may indicate an compensatory mechanisms involving visual networks.

In our study, fALFF was increased in left AG at traditional frequency band. Left AG located in the posterior part of left inferior parietal lobule (IPL), make functional contribution to visual word reading and comprehension (Seghier, 2013; Sliwinska, James, \& Devlin, 2015). On a neurological function level, reading requires several brain regions to work together to recognize a visual input, and correlate to its 
corresponding meaning and sound. Whether distorted or blurred vision caused by AMD involves the visual word recognition processing need to be further studied.

Increased fALFF in left median cingulate gyrus was detected in our result. Study suggested midcingulate cortex (MCC) has contributions to decision making and cognitive control (Holroyd, Ribas-Fernandes, Shahnazian, Silvetti, \& Verguts, 2018). During pain processing, MCC received nociceptive information from parietal areas, then guide multisensory control (such as eye or body orientation) and reflexive movements (Vogt, 2016). We speculate that loss of visual information caused by visual impairment in AMD patients affect this decision making processing. However, the mechanism of MCC function remain elusive and is worthy of further discussion.

\section{Frequency-Dependent Effect}

In current study, we investigated changes of LFFs amplitudes between AMD and HC groups at three different frequency bands (traditional frequency band, slow-4 frequency band and slow- 5 frequency band), and found that the abnormalities of intrinsic brain activity detecting by fALFF in patients with AMD were associated with specific frequency band. Specifically, at slow- 4 frequency band, three different brain regions with abnormal fALFF including fusiform gyrus, supramarginal gyrus and middle cingulate gyrus were observed, whereas at the slow- 5 frequency band, only PCu was found. In addition, fusiform gyrus and supramarginal gyrus with abnormal fALFF at slow-4 band were not found at traditional frequency band. Thus, we conclude that abnormal spontaneous neural activity in fALFF in AMD patient might be more sensitive to the slow- 4 frequency band than to slow- 5 frequency band in some brain regions.

Frequency-dependent effect in specific regions was widely revealed in different diseases including attention-deficit hyperactivity disorder (ADHD) (Di Martino et al., 2008), aMCl (Han et al., 2011), schizophrenia (Hoptman et al., 2010), and depression (Zhou et al., 2020). Di Martino et al. showed fluctuations in slow-4 band provide greater diagnostic information than other bands for children with ADHD (Di Martino et al., 2008). Hoptman et al. revealed that widespread abnormal ALFF/fALFF at slow-4 frequency band was observed in schizophrenia patients (Hoptman et al., 2010). Consistent with our results, these two findings also indicate that slow-4 frequency band is more sensitive in detecting LFFs changes. Thus, the existence of sensitive frequency bands should be taken into consideration when measuring the LFFs changes of brain activity in AMD patients in further study.

Indeed, the brain regions at slow- 4 frequency band and slow- 5 frequency band showed a little different from which at traditional frequency band when ALFF or PerAF were calculated, but the brain regions with abnormal ALFF or PerAF measured at the slow- 4 or slow- 5 frequency band are either included in the traditional frequency band or adjacent to the brain regions measured at the traditional frequency band. In this case, no frequency-dependent effect was observed in ALFF or PerAF in our results. From a methodological point of view, the possible explanation is that fALFF might have higher specificity and sensitivity of detection LFFs than ALFF and PerAF (Zuo et al., 2010). However, the specific neural mechanisms of each frequency band have not been fully explained yet. Researchers suggested neuronal 
oscillations are associated with the activity of chemical synaptic signaling, input selection, and neuron plasticity (Buzsáki \& Draguhn, 2004). Further studies are needed to examine whether such frequencydependent effect could be applied for disease diagnosis or monitoring AMD progression.

This study has some potential limitations. Firstly, the sample size of our study is relatively small. Studies with larger sample sizes are needed to further analyze and confirm these results. Secondly, it is a crosssectional study in AMD patients. Patients with different stages of AMD may exhibit different patterns of brain activity alterations. The patients with AMD should be categorized into different disease stages to explore LFFs changes at different frequency bands. Thirdly, MMSE and MoCA tests of cognitive ability including tasks that relied on visual ability or involved visual cueing are unsuitable for patients with vision impairment. The validated cognitive tests would help us to detect the difference in cognitive level between AMD and HCs.

\section{Conclusions}

To our knowledge, it is the first study to report that abnormal LFFs amplitudes occur in patients with AMD. It showed significant difference in several brain region in ALFF, fALFF and PerAF, respectively at three frequency bands between AMD patients and HCs. The abnormal spontaneous neural activity in fALFF in AMD patients is possibly frequency-dependent in specific brain regions. This study expands our understanding of the potential influence of visual impairment on neural activity in specific brain regions and may provide a new perspective to explain the pathological mechanism of AMD.

\section{Declarations}

\section{Acknowledgements}

We thank all the patients who participated in this study, as well as the colleagues who contributed in the research paper.

\section{Funding Sources}

This work is supported by grants from National Natural Science Foundation of China [No 81871325], Project of STCSM [20Y11911800] and Project of Chongming STCSM [CKY2021-41].

\section{Ethical Approval}

Ethical approval was given by Shanghai Tenth People's Hospital's medical ethics committee in line with the Declaration of Helsinki.

\section{Consent to Participate}

All participants signed informed consents after informing of the purposes, contents, and potential risks. 


\section{Consent to Publish}

All participants in this study consent to publish their data..

\section{Conflict of Interest}

None of the authors have a conflict of interest to declare.

\section{Availability of data and material}

The datasets generated and analyzed during the current study are not publicly available due the fact that they constitute an excerpt of research in progress but are available from the corresponding author on reasonable request.

\section{Author Contributions}

Author contributions included conception and study design (GYT and QP), data collection or acquisition (QP, FJX and PL), statistical analysis (FJX and XLS), interpretation of results (GYT, FJX, XLS and QP), drafting the manuscript work or revising it critically for important intellectual content (All authors) and approval of final version to be published and agreement to be accountable for the integrity and accuracy of all aspects of the work (All authors).

\section{References}

1. Augustin, A., Sahel, J. A., Bandello, F., Dardennes, R., Maurel, F., Negrini, C. ... Berdeaux, G. (2007). Anxiety and depression prevalence rates in age-related macular degeneration. Invest Ophthalmol Vis Sci, 48(4), 1498-1503. doi:10.1167/iovs.06-0761

2. Ballmaier, M., Toga, A. W., Blanton, R. E., Sowell, E. R., Lavretsky, H., Peterson, J. ... Kumar, A. (2004). Anterior cingulate, gyrus rectus, and orbitofrontal abnormalities in elderly depressed patients: an MRIbased parcellation of the prefrontal cortex. Am J Psychiatry, 161(1), 99-108. doi:10.1176/appi.ajp.161.1.99

3. Biswal, B., Yetkin, F. Z., Haughton, V. M., \& Hyde, J. S. (1995). Functional connectivity in the motor cortex of resting human brain using echo-planar MRI. Magn Reson Med, 34(4), 537-541. doi:10.1002/mrm.1910340409

4. Blasiak, J. (2020). Senescence in the pathogenesis of age-related macular degeneration. Cell Mol Life Sci, 77(5), 789-805. doi:10.1007/s00018-019-03420-x

5. Buzsáki, G., \& Draguhn, A. (2004). Neuronal oscillations in cortical networks. Science, 304(5679), 1926-1929. doi:10.1126/science.1099745

6. Caspers, J., Palomero-Gallagher, N., Caspers, S., Schleicher, A., Amunts, K., \& Zilles, K. (2015). Receptor architecture of visual areas in the face and word-form recognition region of the posterior fusiform gyrus. Brain Struct Funct, 220(1), 205-219. doi:10.1007/s00429-013-0646-z 
7. Casten, R., \& Rovner, B. (2008). Depression in Age-Related Macular Degeneration. J Vis Impair Blind, 102(10), 591-599

8. Casten, R. J., Rovner, B. W., \& Tasman, W. (2004). Age-related macular degeneration and depression: a review of recent research. Curr Opin Ophthalmol, 15(3), 181-183. doi:10.1097/01.icu.0000120710.35941.3f

9. Cavanna, A. E., \& Trimble, M. R. (2006). The precuneus: a review of its functional anatomy and behavioural correlates. Brain, 129(Pt 3), 564-583. doi:10.1093/brain/awl004

10. Dawson, S. R., Mallen, C. D., Gouldstone, M. B., Yarham, R., \& Mansell, G. (2014). The prevalence of anxiety and depression in people with age-related macular degeneration: a systematic review of observational study data. BMC Ophthalmol, 14, 78. doi:10.1186/1471-2415-14-78

11. Di Martino, A., Ghaffari, M., Curchack, J., Reiss, P., Hyde, C., Vannucci, M. ... Castellanos, F. X. (2008). Decomposing intra-subject variability in children with attention-deficit/hyperactivity disorder. Biol Psychiatry, 64(7), 607-614. doi:10.1016/j.biopsych.2008.03.008

12. Frings, L., Wagner, K., Quiske, A., Schwarzwald, R., Spreer, J., Halsband, U., \& Schulze-Bonhage, A. (2006). Precuneus is involved in allocentric spatial location encoding and recognition. Exp Brain Res, 173(4), 661-672. doi:10.1007/s00221-006-0408-8

13. Friston, K. J., Williams, S., Howard, R., Frackowiak, R. S., \& Turner, R. (1996). Movement-related effects in fMRI time-series. Magn Reson Med, 35(3), 346-355. doi:10.1002/mrm.1910350312

14. Gehrs, K. M., Anderson, D. H., Johnson, L. V., \& Hageman, G. S. (2006). Age-related macular degeneration-emerging pathogenetic and therapeutic concepts. Ann Med, 38(7), 450-471. doi:10.1080/07853890600946724

15. Han, Y., Wang, J., Zhao, Z., Min, B., Lu, J., Li, K. ... Jia, J. (2011). Frequency-dependent changes in the amplitude of low-frequency fluctuations in amnestic mild cognitive impairment: a resting-state fMRI study. Neuroimage, 55(1), 287-295. doi:10.1016/j.neuroimage.2010.11.059

16. Holroyd, C. B., Ribas-Fernandes, J. J. F., Shahnazian, D., Silvetti, M., \& Verguts, T. (2018). Human midcingulate cortex encodes distributed representations of task progress. Proc Natl Acad Sci USA, 115(25), 6398-6403. doi:10.1073/pnas.1803650115

17. Hoptman, M. J., Zuo, X. N., Butler, P. D., Javitt, D. C., Angelo, D., Mauro, D., C. J., \& Milham, M. P. (2010). Amplitude of low-frequency oscillations in schizophrenia: a resting state fMRI study. Schizophr Res, 117(1), 13-20. doi:10.1016/j.schres.2009.09.030

18. Hu, J. Y., Shu, H. Y., Li, Q. Y., Wu, S. N., Liang, R. B., Ge, Q. M. ... Shao, Y. (2021). Alternation of brain intrinsic activity in patients with hypertensive retinopathy: a resting-state fMRI study. Aging (Albany NY), 13(17), 21659-21670. doi:10.18632/aging.203510

19. Huang, X., Cai, F. Q., Hu, P. H., Zhong, Y. L., Zhang, Y., Wei, R. ... Shao, Y. (2015). Disturbed spontaneous brain-activity pattern in patients with optic neuritis using amplitude of low-frequency fluctuation: a functional magnetic resonance imaging study. Neuropsychiatr Dis Treat, 11, 30753083. doi:10.2147/ndt.S92497 
20. Jia, X. Z., Sun, J. W., Ji, G. J., Liao, W., Lv, Y. T., Wang, J. ... Zang, Y. F. (2020). Percent amplitude of fluctuation: A simple measure for resting-state fMRI signal at single voxel level. PLoS One, 15(1), e0227021. doi:10.1371/journal.pone.0227021

21. Kaarniranta, K., Salminen, A., Haapasalo, A., Soininen, H., \& Hiltunen, M. (2011). Age-related macular degeneration (AMD): Alzheimer's disease in the eye? J Alzheimers Dis, 24(4), 615-631. doi:10.3233/jad-2011-101908

22. Lee, C. S., Larson, E. B., Gibbons, L. E., Lee, A. Y., McCurry, S. M., Bowen, J. D. ... Crane, P. K. (2019). Associations between recent and established ophthalmic conditions and risk of Alzheimer's disease. Alzheimers Dement, 15(1), 34-41. doi:10.1016/j.jalz.2018.06.2856

23. Liu, X., Wang, S., Zhang, X., Wang, Z., Tian, X., \& He, Y. (2014). Abnormal amplitude of low-frequency fluctuations of intrinsic brain activity in Alzheimer's disease. J Alzheimers Dis, 40(2), 387-397. doi:10.3233/jad-131322

24. Logothetis, N. K., Pauls, J., Augath, M., Trinath, T., \& Oeltermann, A. (2001). Neurophysiological investigation of the basis of the fMRI signal. Nature, 412(6843), 150-157. doi:10.1038/35084005

25. Mak, L. E., Minuzzi, L., MacQueen, G., Hall, G., Kennedy, S. H., \& Milev, R. (2017). The Default Mode Network in Healthy Individuals: A Systematic Review and Meta-Analysis. Brain Connect, 7(1), 25-33. doi:10.1089/brain.2016.0438

26. Min, Y. L., Su, T., Shu, Y. Q., Liu, W. F., Chen, L. L., Shi, W. Q. ... Shao, Y. (2018). Altered spontaneous brain activity patterns in strabismus with amblyopia patients using amplitude of low-frequency fluctuation: a resting-state fMRI study. Neuropsychiatr Dis Treat, 14, 2351-2359. doi:10.2147/ndt.S171462

27. Peng, Z. Y., Liu, Y. X., Li, B., Ge, Q. M., Liang, R. B., Li, Q. Y. ... Shao, Y. (2021). Altered spontaneous brain activity patterns in patients with neovascular glaucoma using amplitude of low-frequency fluctuations: A functional magnetic resonance imaging study. Brain Behav, 11(3), e02018. doi:10.1002/brb3.2018

28. Penttonen, M., \& Buzsáki, G. (2003). Natural logarithmic relationship between brain oscillators. Thalamus \& Related Systems, 2(2), 145-152. doi:10.1017/S1472928803000074

29. Puig, J., Blasco, G., Alberich-Bayarri, A., Schlaug, G., Deco, G., Biarnes, C. ... Pedraza, S. (2018). Resting-State Functional Connectivity Magnetic Resonance Imaging and Outcome After Acute Stroke. Stroke, 49(10), 2353-2360. doi:10.1161/strokeaha.118.021319

30. Raichle, M. E., \& Gusnard, D. A. (2005). Intrinsic brain activity sets the stage for expression of motivated behavior. J Comp Neurol, 493(1), 167-176. doi:10.1002/cne.20752

31. Seghier, M. L. (2013). The angular gyrus: multiple functions and multiple subdivisions. Neuroscientist, 19(1), 43-61. doi:10.1177/1073858412440596

32. Sheline, Y. I., \& Raichle, M. E. (2013). Resting state functional connectivity in preclinical Alzheimer's disease. Biol Psychiatry, 74(5), 340-347. doi:10.1016/j.biopsych.2012.11.028

33. Shi, W. Q., Wu, W., Ye, L., Jiang, N., Liu, W. F., Shu, Y. Q. ... Shao, Y. (2019). Altered spontaneous brain activity patterns in patients with corneal ulcer using amplitude of low-frequency fluctuation: An fMRI 
study. Exp Ther Med, 18(1), 125-132. doi:10.3892/etm.2019.7550

34. Sliwinska, M. W., James, A., \& Devlin, J. T. (2015). Inferior parietal lobule contributions to visual word recognition. J Cogn Neurosci, 27(3), 593-604. doi:10.1162/jocn_a_00721

35. van Leeuwen, R., Klaver, C. C., Vingerling, J. R., Hofman, A., \& de Jong, P. T. (2003). Epidemiology of age-related maculopathy: a review. Eur J Epidemiol, 18(9), 845-854. doi:10.1023/a:1025643303914

36. Vogt, B. A. (2016). Midcingulate cortex: Structure, connections, homologies, functions and diseases. J Chem Neuroanat, 74, 28-46. doi:10.1016/j.jchemneu.2016.01.010

37. Whitson, H. E., Chou, Y. H., Potter, G. G., Diaz, M. T., Chen, N. K., Lad, E. M. ... Madden, D. J. (2015). Phonemic fluency and brain connectivity in age-related macular degeneration: a pilot study. Brain Connect, 5(2), 126-135. doi:10.1089/brain.2014.0277

38. Workman, C. I., Lythe, K. E., McKie, S., Moll, J., Gethin, J. A., Deakin, J. F. ... Zahn, R. (2017). A novel resting-state functional magnetic resonance imaging signature of resilience to recurrent depression. Psychol Med, 47(4), 597-607. doi:10.1017/s0033291716002567

39. Wu, Y. Y., Yuan, Q., Li, B., Lin, Q., Zhu, P. W., Min, Y. L. ... Shao, Y. (2019). Altered spontaneous brain activity patterns in patients with retinal vein occlusion indicated by the amplitude of low-frequency fluctuation: A functional magnetic resonance imaging study. Exp Ther Med, 18(3), 2063-2071. doi:10.3892/etm.2019.7770

40. Yu, R., Chien, Y. L., Wang, H. L., Liu, C. M., Liu, C. C., Hwang, T. J. ... Tseng, W. Y. (2014). Frequencyspecific alternations in the amplitude of low-frequency fluctuations in schizophrenia. Hum Brain Mapp, 35(2), 627-637. doi:10.1002/hbm.22203

41. Yu, S., Shen, Z., Lai, R., Feng, F., Guo, B., Wang, Z. ... Gong, L. (2018). The Orbitofrontal Cortex Gray Matter Is Associated With the Interaction Between Insomnia and Depression. Front Psychiatry, 9, 651. doi:10.3389/fpsyt.2018.00651

42. Yue, Y., Jia, X., Hou, Z., Zang, Y., \& Yuan, Y. (2015). Frequency-dependent amplitude alterations of resting-state spontaneous fluctuations in late-onset depression. Biomed Res Int, 2015, 505479. doi:10.1155/2015/505479

43. Zang, Y. F., He, Y., Zhu, C. Z., Cao, Q. J., Sui, M. Q., Liang, M. ... Wang, Y. F. (2007). Altered baseline brain activity in children with ADHD revealed by resting-state functional MRI. Brain Dev, 29(2), 83-91. doi:10.1016/j.braindev.2006.07.002

44. Zhang, D., \& Raichle, M. E. (2010). Disease and the brain's dark energy. Nat Rev Neurol, 6(1), $15-28$. doi:10.1038/nrneurol.2009.198

45. Zhang, S., Qiu, Q., Qian, S., Lin, X., Yan, F., Sun, L. ... Li, X. (2021). Determining Appropriate Screening Tools and Cutoffs for Cognitive Impairment in the Chinese Elderly. Front Psychiatry, 12, 773281. doi:10.3389/fpsyt.2021.773281

46. Zhao, N., Yuan, L. X., Jia, X. Z., Zhou, X. F., Deng, X. P., He, H. J. ... Zang, Y. F. (2018). Intra- and InterScanner Reliability of Voxel-Wise Whole-Brain Analytic Metrics for Resting State fMRI. Front Neuroinform, 12, 54. doi:10.3389/fninf.2018.00054 
47. Zhou, J., Ma, X., Li, C., Liao, A., Yang, Z., Ren, H. ... Chen, X. (2020). Frequency-Specific Changes in the Fractional Amplitude of the Low-Frequency Fluctuations in the Default Mode Network in MedicationFree Patients With Bipolar II Depression: A Longitudinal Functional MRI Study. Front Psychiatry, 11, 574819. doi:10.3389/fpsyt.2020.574819

48. Zhuang, J., Madden, D. J., Duong-Fernandez, X., Chen, N. K., Cousins, S. W., Potter, G. G. ... Whitson, H. E. (2018). Language processing in age-related macular degeneration associated with unique functional connectivity signatures in the right hemisphere. Neurobiol Aging, 63, 65-74. doi:10.1016/j.neurobiolaging.2017.11.003

49. Zou, Q. H., Zhu, C. Z., Yang, Y., Zuo, X. N., Long, X. Y., Cao, Q. J. ... Zang, Y. F. (2008). An improved approach to detection of amplitude of low-frequency fluctuation (ALFF) for resting-state fMRI: fractional ALFF. J Neurosci Methods, 172(1), 137-141. doi:10.1016/j.jneumeth.2008.04.012

50. Zuo, X. N., Di Martino, A., Kelly, C., Shehzad, Z. E., Gee, D. G., Klein, D. F. ... Milham, M. P. (2010). The oscillating brain: complex and reliable. Neuroimage, 49(2), 1432-1445. doi:10.1016/j.neuroimage.2009.09.037

\section{Figures}



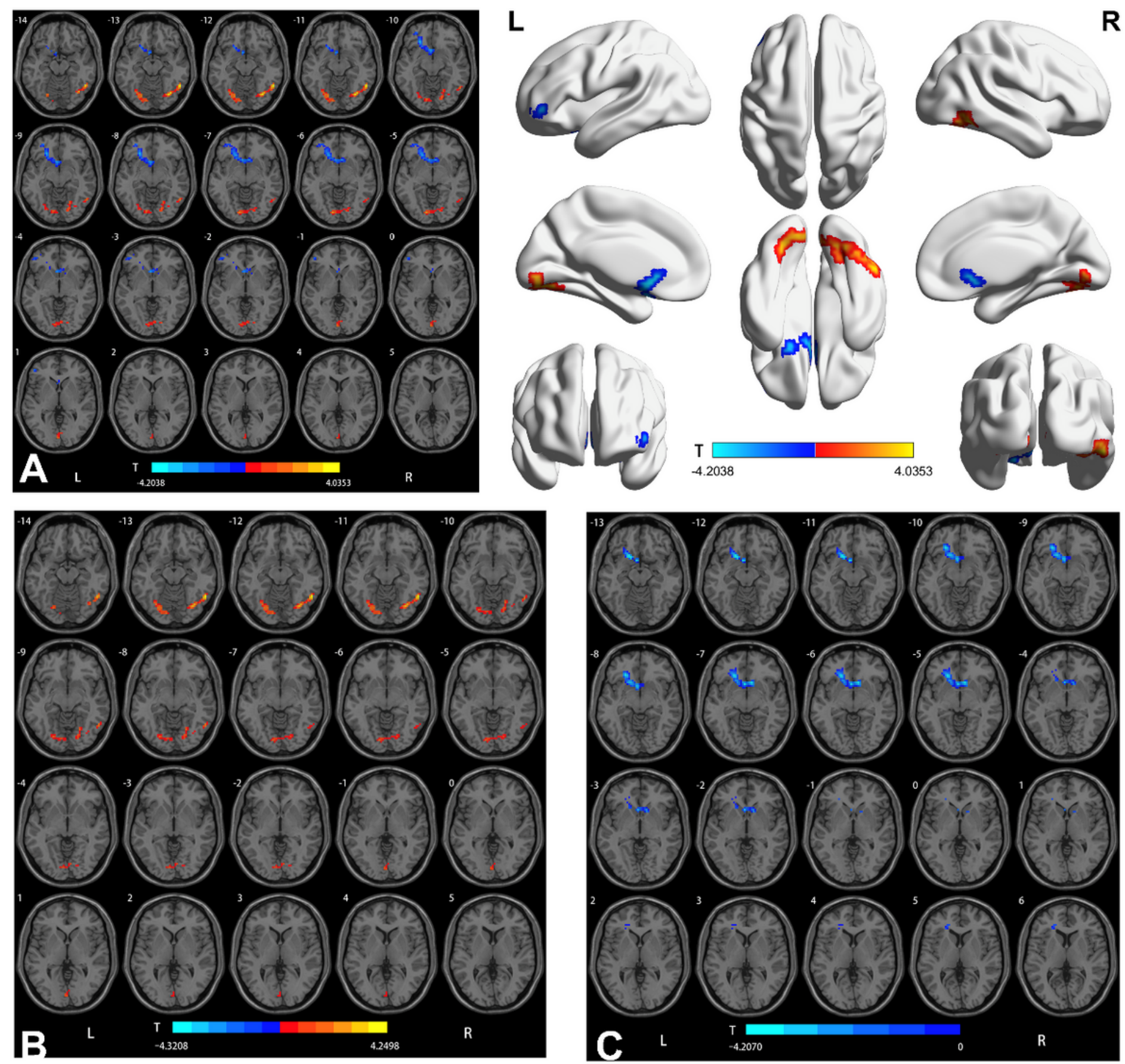

Figure 1

ALFF differences between AMD group and HCs at traditional frequency band (A), slow-4 frequency band (B) and slow-5 frequency band (C).The red and yellow regions represent increased ALFF values in AMD group, compared to HCs. The blue regions represent decreased ALFF values in AMD group, compared to HCs.

Abbreviations: ALFF, amplitude of low-frequency fluctuation; AMD, Age-related macular degeneration; HCs, healthy controls; R, right; L, left; 

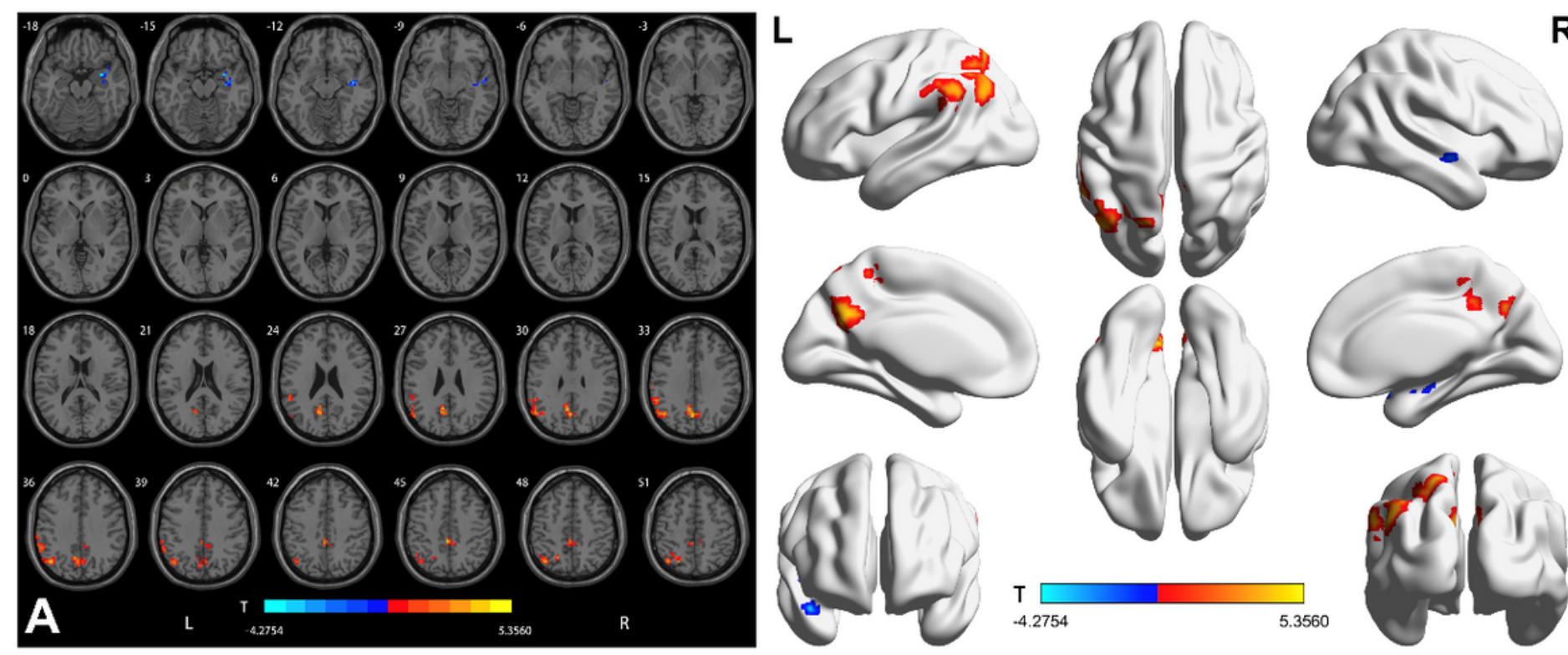

$\mathbf{R}$
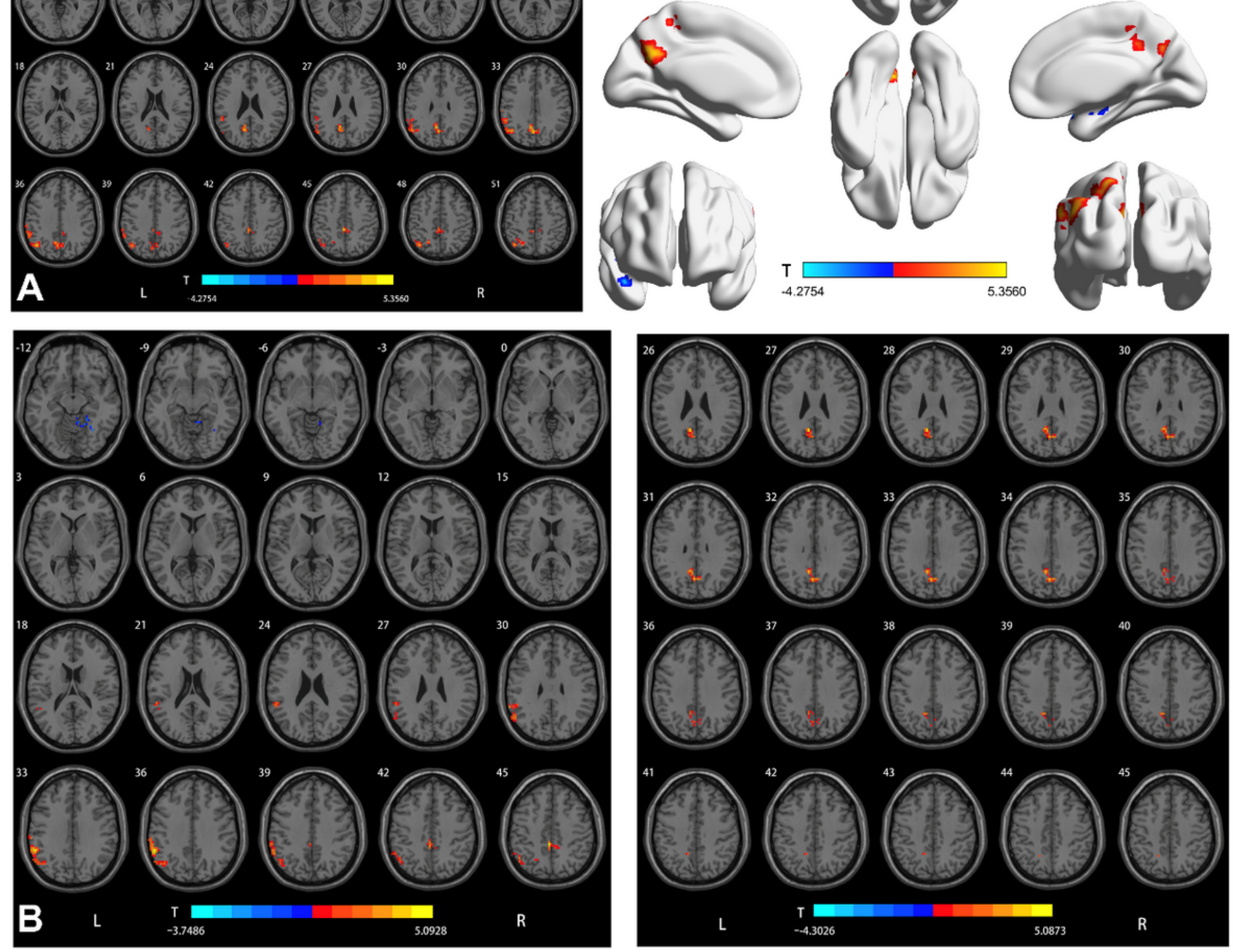

Figure 2

fALFF differences between AMD group and HCs at traditional frequency band (A), slow- 4 frequency band (B) and slow-5 frequency band (C). The red and yellow regions represent increased fALFF values in AMD group, compared to HCs. The blue regions represent decreased fALFF values in AMD group, compared to HCs.

Abbreviations: fALFF, fractional amplitude of low-frequency fluctuation; AMD, Age-related macular degeneration; $\mathrm{HCs}$, healthy controls; $\mathrm{R}$, right; $\mathrm{L}$, left; 

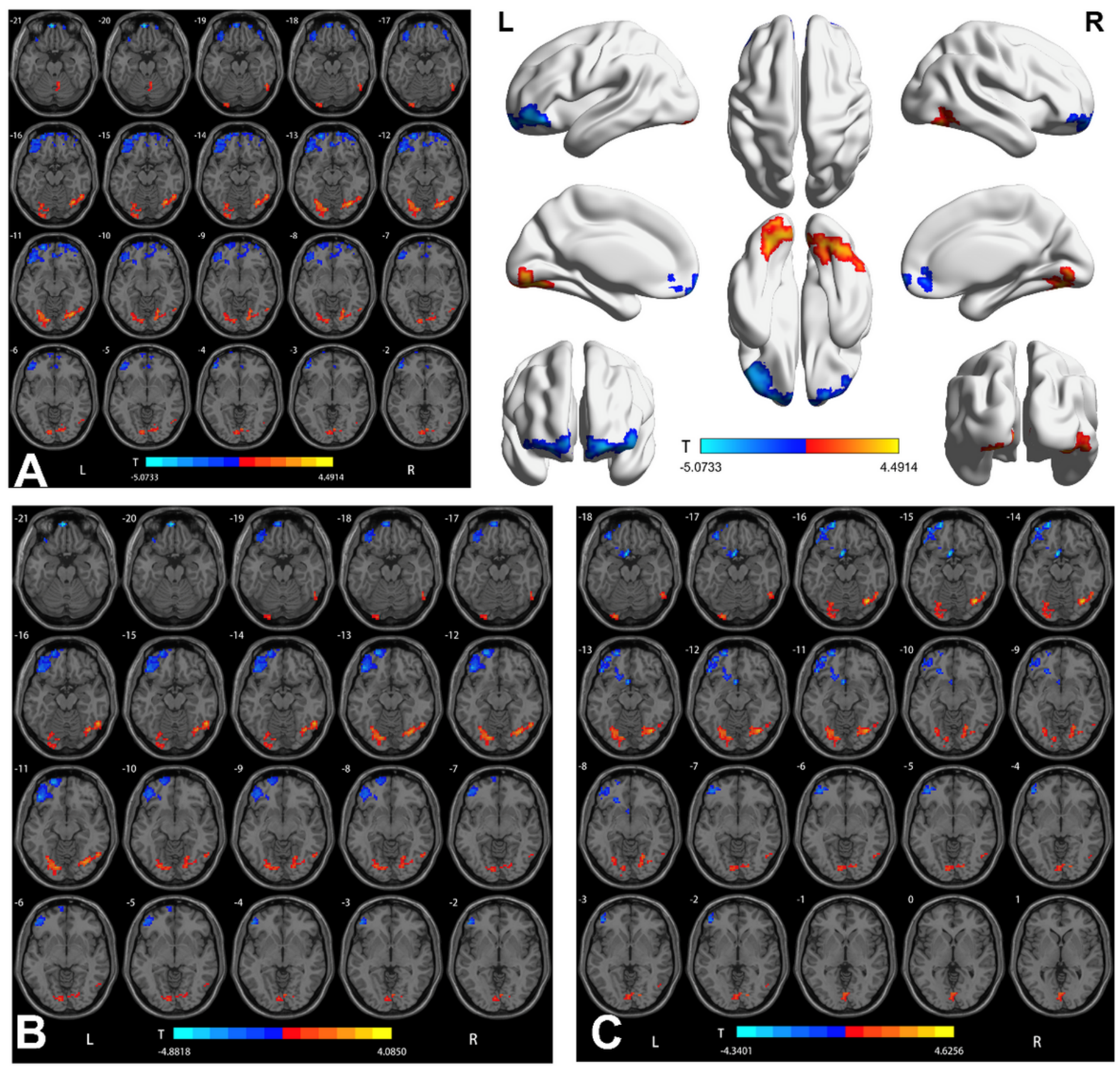

Figure 3

PerAF differences between AMD group and HCs at traditional frequency band (A), slow-4 frequency band (B) and slow-5 frequency band (C). The red and yellow regions represent increased PerAF values in AMD group, compared to HCs. The blue regions represent decreased PerAF values in AMD group, compared to HCs.

Abbreviations: PerAF, percent amplitude of fluctuation; AMD, Age-related macular degeneration; HCs, healthy controls; R, right; L, left. 


\section{Supplementary Files}

This is a list of supplementary files associated with this preprint. Click to download.

- AuthorChecklist.pdf 